\title{
The autonomic signatures of epilepsy: diagnostic clues and novel treatment avenues
}

\author{
Roland D. Thijs ${ }^{1,2}$
}

Received: 12 March 2019 / Accepted: 14 March 2019 / Published online: 22 March 2019

(c) Springer-Verlag GmbH Germany, part of Springer Nature 2019

The epileptic networks are intimately connected with the autonomic nervous system (ANS) as exemplified by a plethora of clinical manifestations [9]. Early case descriptions in people in epilepsy have helped to unveil the hidden ties between heart and brain. As a prelude to the discovery of the central autonomic network (CAN), Russell noted well over 100 years ago that seizures cannot only increase but also decrease heart rate: "He uttered a cry and was seen to be rubbing his hands together. His pulse was immediately examined for but was not palpable" [8]. The paroxysmal disruptions of normal brain activity seen in epilepsy appeared an excellent model to explore the brain-heart axis. Since Russell's observations significant progress has been made: functional studies have helped to unravel the CAN structures and more recent studies hallmark the translation of autonomic concepts into clinical applications.

This issue of Clinical Autonomic Research includes a series of articles highlighting the various clinical implications of ANS involvement in epilepsy. Baumgartner and colleagues provide a comprehensive review of the full spectrum of autonomic symptom and signs in epilepsy [3]. Seizures may affect various autonomic parameters, but cardiovascular manifestations seem to be the most prominent expression. Sympathetic responses predominate during most seizures, causing tachycardia, tachypnea, increased blood pressure, pupillary dilatation, diaphoresis and facial flushing. Autonomic symptoms can provide important clues to identify the seizure onset zone. Autonomic symptoms are generally more prominent when they originate from the temporal lobe, thus demonstrating the importance of the limbic structures to the CAN. Ictal onset tachycardia is a very frequent sign,

Roland D. Thijs

rthijs@sein.nl

1 Stichting Epilepsie Instellingen Nederland (SEIN), Heemstede, The Netherlands

2 Department of Neurology, Leiden University Medical Centre, Leiden, The Netherlands with prevalence rates ranging from 80 to $100 \%$. Ictal tachycardia is more prevalent in temporal than in extratemporal lobe seizures and is a hallmark of convulsive seizures (i.e. focal to bilateral tonic-clonic seizures as well as generalised tonic-clonic seizures). The preponderance of temporal lobe seizures applies to most other ictal autonomic manifestations. The presence, type and severity of the autonomic features are determined not only by the localisation of the seizure onset zone, but also by the lateralisation, seizure propagation, timing (ictal vs. postictal) and presence of structural ANS changes (e.g. decreased heart rate variability) (Fig. 1). The presence of autonomic seizures may sometimes help in determining an underlying cause, as ictal autonomic features, in particular piloerection, seem to be more prevalent in limbic encephalitis [3]. Ictal autonomic signs can be extremely subtle and require detailed recordings to prove the epileptic nature: Toth and colleagues present an interesting case where they convincingly demonstrate that anterior cingulate seizures may manifest as arousals from sleep [10].

Peri-ictal autonomic changes may not only help to improve diagnostic work-up but also to elucidate the pathophysiology of a devastating complication of epilepsy: sudden unexpected death in epilepsy (SUDEP). Barot and Nei review the autonomic aspects of SUDEP [2]. SUDEP is highly linked to seizures, with most deaths occurring after nocturnal convulsive seizures. Fatal seizures are characterised by postictal generalised electroencephalograpic (EEG) suppression and transient episodes of apnea and bradycardia culminating in a fatal asystole. Evidence is mounting that the fatal event is caused by excessive inhibition that in turn causes depression of brainstem activity and hereby cessation of vital signs. An interesting prospect is the rapid development of wearable, non-EEG-based seizure detection devices that might alert carers to seizures that could otherwise go unnoticed. This is critically important as SUDEP is mostly unwitnessed, and nocturnal supervision appears to decrease SUDEP risk [11]. Adequate seizure detection may have the 


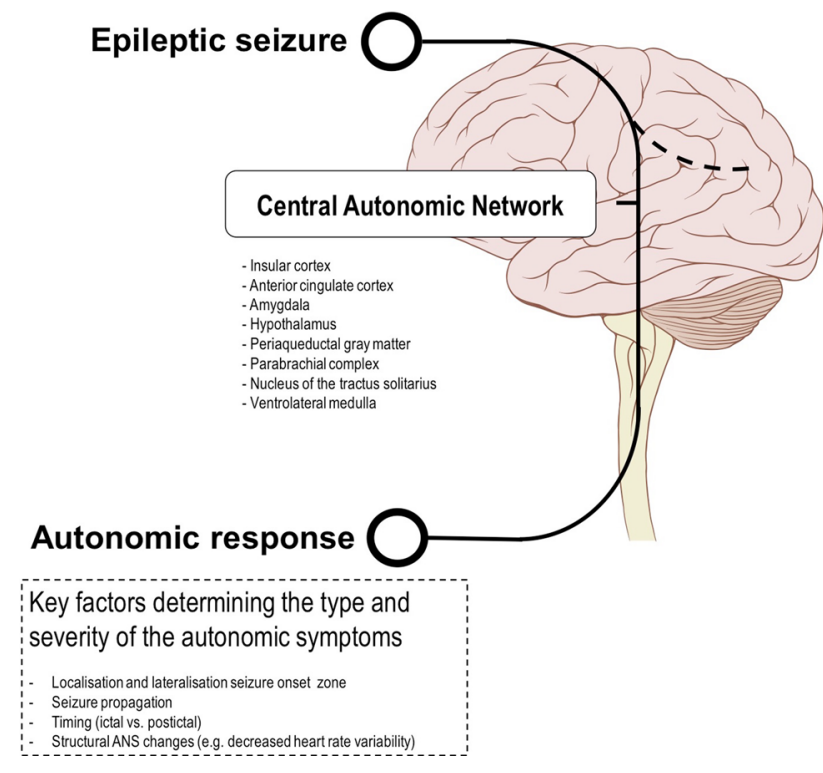

Fig. 1 Interaction between epileptic seizures and autonomic symptoms. ANS Autonomic nervous system. Slightly modified from illustration by Patrick J. Lynch: skull and brain normal human.svg (commons.wikimedia.org/wiki/media/File:Brain_stem_normal_ human.svg)

potential to minimise these complications and to ameliorate treatment evaluation as seizures, particularly those at night, are often underreported [4].

van Westrhenen et al. [12] performed a literature review of the seizure detection algorithms using autonomic function alterations. Various autonomic signals, including cardiovascular, respiratory and transpiration changes, can be used to detect seizures. Autonomic changes are considered to be attractive parameters for seizure detection as the autonomic alterations can precede ictal EEG discharges by several seconds. Unimodal autonomic algorithms appear to be sensitive predictors of seizure, but false alarms rates are still high. Multimodal algorithms appear to be an attractive strategy to reduce the false alarm rates, but further studies are needed. Interestingly, a recent prospective long-term trial using a multimodal algorithm (heart rate and accelerometry) indicated that this approach can be fruitful (mean sensitivity per participant $86 \%$; false alarm rate 0.03 per night) [1].

Epilepsy may also cause functional ANS changes, including decrease of heart rate variability [9]. Liu and colleagues report another approach to quantify interictal ANS dysfunction of the deceleration and acceleration capacity of the heart rate [6]. In this study, like heart rate variability (HRV), both acceleration and deceleration capacity were impaired in people with drug refractory epilepsy compared to healthy controls, with the lowest values occurring during the night. Deceleration capacity appeared to have the greatest discriminatory power to differentiate patients from controls. The phase-rectified signal averaging method may serve as a complementary approach to characterise ANS dysfunction in epilepsy.

The vagal nerve is a critical relay connecting the CAN and the vital organs and an attractive structure to modulate during the treatment of epilepsy. Garamendi-Ruiz and Gomez-Esteban review the cardiovascular aspects of vagal nerve stimulation (VNS) [5]. Long-term stimulation data in epilepsy indicate that VNS causes only subtle or no significant changes in HRV and blood pressure. Indeed, VNS might reduce SUDEP risk. Autonomic biofeedback therapy is another example of how ascending autonomic pathways can be used to modulate the cortical epileptic networks [7]. Evidence is accumulating on the efficacy of biofeedback using galvanic skin response in treating epileptic seizures.

In summary, the autonomic seismograph is still under construction. The localising and lateralising value of autonomic manifestations could be improved if the broader clinical context is put in the equation and the timing related to coincidence of other symptoms. We still fail to reliably predict SUDEP: postictal autonomic measures or markers of ANS dysfunction, including interictal HRV acceleration and deceleration capacity, might proof fruitful to improve SUDEP risk prediction. Seizure detection is a promising intervention in those with refractory epilepsy with signs of missed seizures. Modulation of the ANS is another promising avenue that might, like VNS, help to partly reduce seizure burden in those with refractory epilepsy.

Roland D. Thijs, MD PhD

Guest Editor

\section{Compliance with ethical standards}

Conflict of interest None.

\section{References}

1. Arends J, Thijs RD, Gutter T, Ungureanu C, Cluitmans P, Van Dijk J, van Andel J, Tan F, de Weerd A, Vledder B, Hofstra W, Lazeron R, van Thiel G, Roes KCB, Leijten F, the Dutch TeleEpilepsy C (2018) Multimodal nocturnal seizure detection in a residential care setting: a long-term prospective trial. Neurology 91:e2010-e2019

2. Barot N, Nei M (2018) Autonomic aspects of sudden unexpected death in epilepsy (SUDEP). Clin Auton Res. https://doi. org/10.1007/s10286-018-0576-1

3. Baumgartner C, Koren J, Britto-Arias M, Schmidt S, Pirker S (2019) Epidemiology and pathophysiology of autonomic seizures: a systematic review. Clin Auton Res. https://doi.org/10.1007/ s10286-019-00596-x

4. Elger CE, Hoppe C (2018) Diagnostic challenges in epilepsy: seizure under-reporting and seizure detection. Lancet Neurol 17:279-288

5. Garamendi-Ruiz I, Gomez-Esteban JC (2017) Cardiovascular autonomic effects of vagus nerve stimulation. Clin Auton Res. https://doi.org/10.1007/s10286-017-0477-8 
6. Liu H, Yang Z, Meng F, Guan Y, Ma Y, Liang S, Lin J, Pan L, Zhao M, Hao H, Luan G, Zhang J, Li L (2018) Deceleration and acceleration capacities of heart rate in patients with drugresistant epilepsy. Clin Auton Res. https://doi.org/10.1007/s1028 6-018-0569-0

7. Nagai Y, Aram J, Koepp M, Lemieux L, Mula M, Critchley H, Sisodiya S, Cercignani M (2018) Epileptic seizures are reduced by autonomic biofeedback therapy through enhancement of frontolimbic connectivity: a controlled trial and neuroimaging study. Ebiomedicine 27:112-122

8. Russell AE (1906) Cessation of the pulse during the onset of epileptic fits, with remarks on the mechanism of fits. Lancet 2:152-154

9. Shmuely S, van der Lende M, Lamberts RJ, Sander JW, Thijs RD (2017) The heart of epilepsy: current views and future concepts. Seizure 44:176-183
10. Toth E, Chaitanya G, Pogwizd M, Pizarro D, Ilyas A, Pogwizd S, Pati S (2018) Dynamics of seizure-induced behavioral and autonomic arousal. Clin Auton Res. https://doi.org/10.1007/s1028 6-018-0543-x

11. van der Lende M, Hesdorffer DC, Sander JW, Thijs RD (2018) Nocturnal supervision and SUDEP risk at different epilepsy care settings. Neurology 91:e1508-e1518

12. van Westrhenen A, De Cooman T, Lazeron RHC, Van Huffel S, Thijs RD (2018) Ictal autonomic changes as a tool for seizure detection: a systematic review. Clin Auton Res. https://doi. org/10.1007/s10286-018-0568-1 